\title{
Editorial
}

\section{Cardiology in the Post-Genomic Era : Road to Personalized Medicine}

\author{
Zahurul A. Bhuiyan
}

(Bangladesh Heart Journal 2017; 32(1) : 1-2)

Synchronous action potential of cardiomyocytes lead to rhythmic contraction of the heart. Cardiac action potential is generated by interplay of wide range of cardiac ion channels e.g. $\mathrm{Na}^{+}, \mathrm{K}^{+}$and $\mathrm{Ca}^{++}$ion channels. ${ }^{1}$ Each ion channel has different functional subunits, which are subdivided in functional domains. Ion channels are supported by different supporting structures, molecules and chaperones, which are required for proper localization of the ion channels and also for their well coordinated function. Cardiac action potentials are propagated from one cardiac cell to its neigbourng cells through the gap junctions located in the intercalated discs. Cardiomyocytes are attached to each other by desmosomal proteins e.g. plakophillin (PKP2), plakoglobin (JUP), desmoglein (DSG2) etc (Bhuiyan et al. 2009). ${ }^{1}$ Cardiac sarcomeric proteins are the contractile machineries, which are located inside the cardiomyocytes ; action-potential generated currents lead to contraction of these sarcomeric machineries. All these components in the heart are encoded by a long list of genes ; widely investigated major genes in this list are cardiac $\mathrm{Na}^{+}$channel encoding SCN5A, $\mathrm{K}^{+}$channel encoding $K C N Q 1$ and $K C N H 2, \mathrm{Ca}^{++}$channel associated genes RYR2, CASQ2, CACNA1C, Calm1, Calm2, Calm3, and desmosomal proteins encoding genes, PKP2, JUP, DSG2, TMEM43 etc. Defect (mutation) in these genes are widely known to cause various hereditary cardiac disorders e.g. long and short QT syndromes, Brugada syndrome, Catecholaminergic polymorphic ventricular tachycardia, Arrhythmogenic right ventricular cardiomyopathy/dysplasia, etc. ${ }^{1}$ Defects in the cardiac sarcomeric and associated genes e.g. MYBPC3, MYH7, TTN are causal to Hypertrophic and/or Dilated cardiomyopathies.

Address of Correspondence: Zahurul A. Bhuiyan, MBBS, Ph.D, FESC, Head of the Molecular Diagnostic Laboratory, Division of Genetic Medicine, University Hospital Lausanne (CHUV), Lausanne, Switzerland. Email : Z.A.Bhuiyan@CHUV.CH
Structural or non-genetic heart diseases are the predominant cardiac diseases. Ischemic cardiopathy, the more prevalent structural heart disease, is predominantly non-genetic in origin, though individual genetic makeup are considered to play a role in ischemic heart diseases and arrhythmias. ${ }^{2}$ Until now these two entities, non-genetic and genetic heart diseases, were kept and dealt separately. Arrhythmias due to genetic defects are causal to specific primary arrhythmias and these diseases are usually familial. ${ }^{1}$ In case of primary familial arrhythmias, a genetic defect is usually found in about $70 \%$ of patients ; treatment usually depends on the involved gene, mutation location and pathology, family history for the disease, history of syncope, etc. ${ }^{1}$ Primary arrhythmia patients usually have no structural heart abnormality : their defect is predominantly in the electrical properties of the heart, which can't be visualized.

But, what we are missing here, is how to deal with a patient who has ischemic heart disease compounded by mutation in a cardiac ion channel gene. Do we know how such a ion channel gene mutation could modify the disease profile in a patient with ischemic heart disease? Mutation in two different genes are also found in 5-10\% of patients with genetic heart diseases, and which could aggravate or worsen the disease. ${ }^{1}$ This is not restricted to disease causal pathogenic mutation only as recent studies showed that individual genetic profile could exacerbate post-ischemic arrhythmias. ${ }^{3} \mathrm{~A}$ team led by Prof. Peter J Schwartz conducted a study on patients admitted with myocardial infarction ; their team searched for common variations (polymorphism) in the cardiac repolarization ion channel gene $\mathrm{KCNH} 2$ and investigated their effect on arrhythmia propensity in the subacute phase of myocardial infarction. ${ }^{3} \mathrm{~K} 897 \mathrm{~T}$ is a common variation or polymorphism in the $\mathrm{KCNH} 2$ gene : $80 \%$ of general population has lysine $(\mathrm{K})$ in the 897 th amino-acid position of the $\mathrm{KCNH} 2$ gene, whereas $20-30 \%$ population has

DOI: http://dx.doi.org/10.3329/bhj.v32i1.34162

Copyright $(2017$ Bangladesh Cardiac Society. Published by Bangladesh Cardiac Society. This is an Open Access articles published under the Creative Commons Attribution-NonCommercial 4.0 International License (CC BY-NC). This license permits use, distribution and reproduction in any medium, provided the original work is properly cited and is not used for commercial purposes. 
threonine $(T)$ instead of lysine $(K)$. They showed that patients with threonine $(\mathrm{T})$ in 897th position are more prone to develop post-ischemic arrhythmias than those with lysine in that position. ${ }^{3}$

We know that $\mathrm{S} 1103 \mathrm{Y}$ is a common variant/polymorphism in SCN5A gene in African poulation : individuals who have tyrosine $(\mathrm{Y})$ in the 1103rd position of SCN5A protein are susceptible to develop arrhythmia which can lead finally to sudden cardiac death. ${ }^{4}$ KCNE1 gene encodes for the b-subunit of repolarization curent $\mathrm{I}_{\mathrm{ks}}$ (encoded by KCNQ1); D85N in KCNE1 is a common variant in Japanese population and individuals with Asparagine $(\mathrm{N})$ at 85th position of KCNE1 gene are susceptible to drug induced arrhythmia. ${ }^{5}$ These studies were conducted mostly in conjunction to genetic heart dieases i.e in patients with primary arrhythmias. But, how would we deal with a patient suffering from potentially dangereous arrhythmias secondary to ischemia or other structural heart diseases? In addition he /she has single or multiple genetic variants that may influence the disease, positively or negatively. What about the genetic variants with a protective effect that rather attenuates or helps to retard developpement of disease pathology ? What about variants in the drug metabolizing genes acting on cardiac cells, what about the genes and variants that work for ATP generation in heart or during adrenaline induced phosphorylation of $\mathrm{I}_{\mathrm{ks}}$ ?

In the post genomic era, human genome sequence is an open book now as we can sequence the genome of a patient in less than a week. Highthrougput gene sequencing is pretty affordable: whole exome sequencing i.e. DNA sequencing of all the known genes present in a man could be done only with 1000 US Dollar. Intepretation of the genomic data is also becoming less tedious. Studies are now required to look at the combined effect of the genetic and non-genetic aspects of the arrrhythmias. In 10 yrs span to time, we will discover many component of genetics that influences the cardiac diseases, their pathology and evolution. Patients with certain genetic profiles are expected to be more arrhythmia prone than others with different genetic profile or reverse. Individual genetic and epigenetic makeup (imprinting) will be evaluated while consulting a patient. Further, at present, drug treatment for arrhythmia is primarily focused to relieve symptoms and lower the risk of sudden cardiac death, or is targeted to few ion channels or receptors; present therapy does not target the molecular basis for arrhythmogenesis in an individual patient.

Precise genetic data will lead to the development of individually adapted, targeted therapy for arryhthmia treatment, focused to personalized needs. One such example is excessive SCN5A derived $\mathrm{Na}^{+}$causing long QT syndrome type 3 : a drug (e.g. Mexiletine) that reduces this excessive $\mathrm{Na}^{+}$in heart would treat the exact pathology and thereby treat the disease by eliminating the pathology process. ${ }^{6}$ Similarly cellular loss or diminution of $\mathrm{K}^{+}$ causes long QT syndrome : a drug that targets the cardiac $\mathrm{K}^{+}$channels and normalizes their property will be effective in treating long QT syndrome caused by reduced $\mathrm{K}^{+}$ current in the heart.

So, it is imperative that we are proceeding toward a new direction in personalized medicine. Individual genetic profile will be necessary to evaluate each patient and his/her disease. All future cardiologists will be required to have an excellent understanding about genetics in their bedside medicine practice. We are on the road to a new era of personalized genomic medicine.

\section{References:}

1. Bhuiyan Z.A. Clinical and genetic spectrum of hereditary cardiac arrhythmia syndromes. Ph.D thesis. University of Amsterdam. ISBN: 9789090243184.

2. Bezzina CR, Pazoki R, Bardai A, Marsman RF, de Jong JS, Blom MT, et al.Genome-wide association study identifies a susceptibility locus at 21q21 for ventricular fibrillation in acute myocardial infarction. Nat Genet. 2010;42:688-91.

3. Crotti L, Hu D, Barajas-Martinez H, De Ferrari GM, Oliva A, Insolia $R$, et al. Torsades de pointes following acute myocardial infarction: Evidence for a deadly link with a common genetic variant. Heart Rhythm. 2012;9:1104-12.

4. Plant LD, Bowers PN, Liu Q, Morgan T, Zhang T, State MW, et al. Common Cardiac Sodium Channel Variant Associated With Sudden Infant Death in African Americans, SCN5A S1103Y. J Clin Invest. 2006; 116: 430-5.

5. Nishio $\mathrm{Y}$, Makiyama T, Itoh $\mathrm{H}$, Sakaguchi T, Ohno S, Gong $Y Z$, et al. D85N, a KCNE1 polymorphism, is a disease-causing gene variant in long QT syndrome. J Am Coll Cardiol. 2009;54:812-9.

6. Mazzanti A, Maragna R, Faragli A, Monteforte N, Bloise R, Memmi M, et al. Gene-Specific Therapy with Mexiletine Reduces Arrhythmic Events in Patients With Long QT Syndrome Type 3. J Am Coll Cardiol. 2016;67:1053-8. 